\title{
ARTIGO
}

\section{GESTÃO DO CAPITAL DE GIRO EM OPERADORAS DE PLANOS DE SAÚDE: UMA ANÁLISE SOB A PERSPECTIVA DO MODELO DINÂMICO ${ }^{1}$}

\author{
Ewerton Alex Avelar ${ }^{2}$ \\ Antônio Artur de Souza
}

\author{
RESUMO
}

O objetivo deste trabalho é apresentar os resultados de uma pesquisa que visou analisar a gestão do capital de giro, sob a perspectiva do modelo dinâmico, das operadoras de planos de saúde (OPSs) brasileiras no período de 2012 a 2016. Tal estudo pode ser classificado como descritivo e quantitativo. Foram coletados dados financeiros anuais de 818 operadoras classificadas pela Agência Nacional de Saúde Suplementar (ANS) nas seguintes modalidades: autogestão, cooperativa médica, cooperativa odontológica, filantropia, medicina de grupo e odontologia de grupo. Os dados foram analisados por meio das técnicas de estatística descritiva e da estatística do qui-quadrado. Os resultados obtidos na pesquisa empírica demonstram que as estruturas financeiras das OPSs, que evidenciam aspectos da gestão de seu capital de giro segundo o modelo dinâmico, são discrepantes entre as diferentes modalidades de operadoras. Tal constatação foi estatisticamente significativa em todos os anos analisados, conforme a estatística do qui-quadrado. Apesar dessa constatação por modalidade, é importante ressaltar que, no geral, todas operadoras apresentaram uma deterioração de suas estruturas financeiras entre os anos de 2015 e 2016: verificou-se um aumento geral na necessidade de capital de giro dessas organizações, reduzindo sua liquidez.

Palavras-chave: Gestão do capital de giro. Modelo dinâmico. Operadoras de planos de saúde. Estrutura financeira.

\section{INTRODUÇÃO}

Diante da competitividade das últimas décadas no ambiente corporativo, geralmente a gestão adequada dos recursos financeiros da empresa passa a ser um importante fator de sobrevivência. Nesse contexto, destaca-se a gestão de capital de giro (GITMAN, 2010). Segundo Ukaegbu (2014), o objetivo da gestão do capital de giro é garantir que a empresa esteja hábil a pagar suas obrigações de curto prazo adequadamente.

Ambrozini et al. (2014) destacam que a análise do capital de giro não deve se restringir à análise estática do capital de giro líquido ou aos indicadores de liquidez tradicionalmente usados em análises de demonstrações financeiras. Nesse sentido, Camargos et al. (2014) ressaltam a importância do emprego do modelo dinâmico (ou modelo Fleuriet), que realça, essencialmente, a análise de contas relacionadas à gestão do capital de giro da empresa.

De acordo com Vieira (2008), a partir dos valores apresentados pelas empresas para cada uma das variáveis básicas do modelo dinâmico, é possível classificar suas estruturas financeiras e compreender melhor sua gestão do capital de giro. Segundo Araújo et al. (2013), com base na classificação das estruturas financeiras, as empresas podem ser categorizadas em: excelentes,

${ }^{1}$ Como citar este artigo: AVELAR, Ewerton Alex; SOUZA, Antônio Artur de. Gestão do capital de giro em operadoras de planos de saúde: uma análise sob a perspectiva do modelo dinâmico. ForScience: revista científica do IFMG, Formiga, v. 6 , n. 1, e00300, 2018.

${ }^{2}$ Autor para correspondência: Ewerton Alex Avelar, UFMG, e-mail: ewertonalexavelar@gmail.com ForSci.: r. cient. IFMG campus Formiga, Formiga, v. 6 , n. 1 , e00300, 2018. 
AVELAR, E. A.; SOUZA, A. A. de. Gestão do capital de giro em operadoras de planos de saúde: uma análise sob a perspectiva do modelo dinâmico

sólidas, insatisfatórias, muito ruins, péssimas ou de alto risco. Contudo, apesar da importância da classificação da estrutura financeira das empresas ao longo do tempo para se analisar a sua gestão do capital de giro, não são encontrados estudos com base nas diferentes modalidades de operadoras de planos de saúde (OPSs) atuantes no Brasil.

De modo geral, pode-se dizer as OPSs são organizações que fornecem um abrangente conjunto de serviços de saúde a seus beneficiários, com base no pagamento mensal de um valor fixo por parte desses (ENGBERG et al., 2004). Essas organizações apresentam uma série de particularidades no contexto brasileiro, sendo classificadas em diferentes modalidades, quais sejam: administradora; cooperativa médica; cooperativa odontológica; autogestão; medicina de grupo; odontologia de grupo; seguradora especializada em saúde; ou filantropia (AGÊNCIA NACIONAL DE SAÚDE SUPLEMENTAR - ANS, 2000). As OPSs são amplamente reguladas pela Agência Nacional de Saúde Suplementar (ANS), sendo seu aspecto econômico-financeiro bastante relevante (SANCOVISCHI et al., 2014).

Diante do exposto, este trabalho tem como objetivo apresentar os resultados de uma pesquisa que visou analisar a gestão do capital de giro, sob a perspectiva do modelo dinâmico, das OPSs brasileiras no período de 2012 a 2016. Para tanto, foram propostos os seguintes objetivos específicos: (a) classificar as estruturas financeiras das OPSs brasileiras no período de estudo proposto; (b) analisar a evolução das estruturas financeiras ao longo do tempo; e (c) identificar as diferenças significativas entre as estruturas financeiras das distintas modalidades de OPSs.

Pesquisas que analisem a gestão financeira das operadoras brasileiras se justificam sob várias perspectivas. Do ponto de vista econômico, Sancovschi et al. (2014) verificaram que a dimensão econômico-financeira é mais importante no que se refere à explicação da intervenção direta da ANS em OPSs. Costa (2008) e Magalhães et al. (2015) também ressaltam a importância das informações econômico-financeira para acompanhamento das operadoras por parte da ANS. Cardoso (2005) destaca que esse acompanhamento só é possível por meio do plano de contas padrão imposto por aquela agência. Souza et al. (2016), contudo, afirmam que a alta heterogeneidade das OPSs brasileiras impacta negativamente na eficácia e a eficiência da gestão financeira dessas organizações no país. De forma mais ampla, Varella e Ceschin (2014) apresentam vários problemas de ordem financeira que são enfrentados pelos principais agentes afetados pela regulação da ANS. De acordo com os referidos autores, as "operadoras acham que a margem é muito pequena e, se bobearem, vão quebrar" (VARELLA; CESCHIN, 2014, p. 42).

Do ponto de vista social, dados da ANS indicam que há quase 50 milhões de beneficiário de planos de saúde no país, ou seja, algo próximo de 25\% da população brasileira (ANS, 2017). Ademais, Varella e Ceschin (2014) destacam que, devido à significativa ascensão social da última década no país, houve um descompasso entre o aumento da população assistida pelos planos de saúde e o crescimento da estrutura das OPSs. E apesar dos altos investimentos em saúde (tanto público quanto privado), aqueles advertem que mesmo que nós "consigamos destinar 
AVELAR, E. A.; SOUZA, A. A. de. Gestão do capital de giro em operadoras de planos de saúde: uma análise sob a perspectiva do modelo dinâmico

mais recursos para a saúde, eles continuarão a ser limitados e insuficientes. Nós temos que fazer mais com o que temos, ou seja, teremos que ser mais eficientes" (VARELLA; CESCHIN, 2004, p. 14).

\section{REVISÃO DA LITERATURA}

De acordo com Ambrozini et al. (2014), a gestão de capital de giro é essencial principalmente em ambientes econômicos como o brasileiro, no qual o acesso a financiamento de curto prazo tende a ser complexo. Padoveze e Benedicto (2014) destacam que o termo capital de giro advém da versão circular do processo operacional de geração de lucros em uma empresa convencional, ou seja: adquirir matéria-prima, produzir, vender e receber, voltar a adquirir matéria-prima, produzir, vender e receber novamente.

Padoveze e Benedicto (2014) ressaltam a importância de técnicas e modelos para a gestão do capital de giro nas empresas. Nesse sentido, Fleuriet (2005) destaca o modelo dinâmico (também conhecido como "modelo Fleuriet”). De acordo com Araújo et al. (2013, p. 314), esse modelo foi desenvolvido "com o intuito de estabelecer uma metodologia mais consistente de gestão e avaliação financeira das empresas brasileiras, sobretudo, para a análise financeira ou do capital de giro [...], com foco [no] Balanço Patrimonial”.

Conforme Fleuriet (2005), para calcular as variáveis do modelo dinâmico, é necessário uma reclassificação de algumas contas das demonstrações financeiras (essencialmente do Balanço Patrimonial). De acordo com Theiss Júnior e Wilhelm (2000), as contas do Balanço Patrimonial devem ser divididas em três diferentes grupos: (a) Operacionais (ou cíclicas) relacionadas com as atividades da empresa, ou seja, vinculadas a seus negócios; (b) Estratégicas - referem-se às movimentações vinculadas à alta direção da empresa; e (c) Táticas (financeiras ou erráticas) - de curto ou curtíssimo prazo, geralmente administradas pela tesouraria da empresa.

Para Fleuriet et al. (2003), a análise a partir do modelo dinâmico depende de três variáveis calculadas a partir das demonstrações financeiras reorganizadas: necessidade de capital de giro (NCG), capital de giro (CDG) e saldo de tesouraria (SDT). Francisco et al. (2012) salientam que a NCG, calculada pela diferença entre o ativo operacional (AO) e o passivo operacional (PO), surge quando as saídas de caixa ocorrem antes das entradas efetivas de caixa, o que cria uma necessidade de recursos. Já o CDG é obtido pela diferença entre o passivo estratégico (PE) e o ativo estratégico (AE) (VIEIRA, 2008) e trata do conjunto dos recursos disponíveis para a empresa financiar suas necessidades operacionais (LIMA et al., 2015). Por sua vez, segundo Theiss Júnior e Wilhelm (2000), o SDT é obtido pela diferença entre o ativo financeiro (AF) e o passivo financeiro (PF), mas também pode ser mensurado pela diferença entre o CDG e a NCG (CAMARGOS et al., 2014). O SDT apresenta a dependência da empresa em relação a instituições financeiras (se negativo) ou seu saldo de liquidez (se positivo). Na Figura 1, 
evidenciam-se as relações entre os conceitos de ativos e passivos e as variáveis do modelo dinâmico.

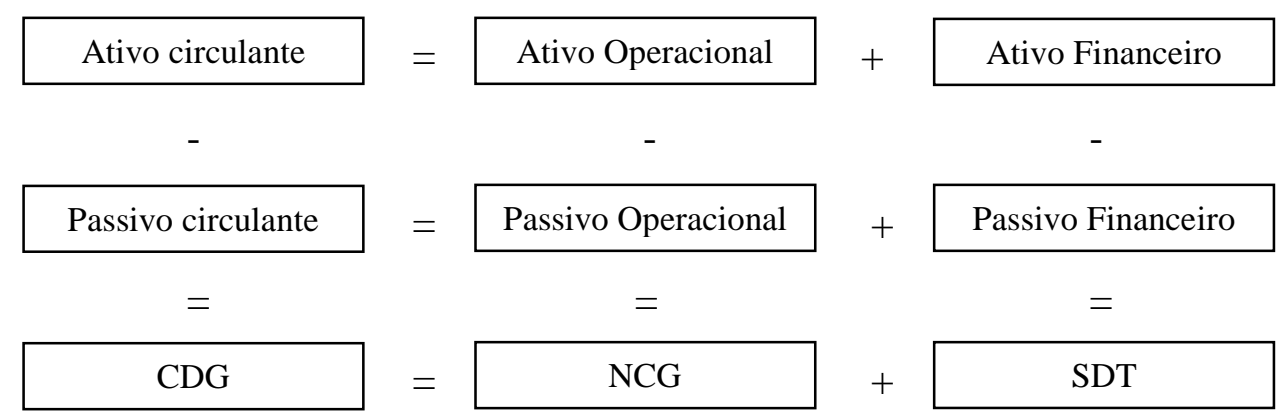

Figura 1 - Relações entre os conceitos e as variáveis do modelo dinâmico Fonte: Adaptado de Braga (1991).

A partir das variáveis supracitadas (CDG, NCG e SDT), Braga (1991) e Marques e Braga (1995) definiram estruturas financeiras de empresas amplamente empregadas em estudos sobre o modelo dinâmico, tais como Theiss Júnior e Wilhelm (2000), Machado et al. (2006), Camargos et al. (2014) e Lima et al. (2015). No Quadro 1, apresenta-se um resumo dos perfis de empresas relativos a suas estruturas financeiras.

\begin{tabular}{|c|c|c|c|c|}
\hline Tipo & CDG & NCG & $\begin{array}{l}\text { SD } \\
\text { T }\end{array}$ & Situação \\
\hline I & + & - & + & Excelente \\
\hline II & + & + & + & Sólida \\
\hline III & + & + & - & Insatisfatória \\
\hline IV & - & + & - & Péssima \\
\hline V & - & - & - & Muito ruim \\
\hline VI & - & - & + & Alto Risco \\
\hline
\end{tabular}

Quadro 1 - Tipos de estrutura a partir das variáveis calculadas do modelo dinâmico Fonte: Adaptado de Braga (1991) e Marques e Braga (1995).

As estruturas financeiras de empresas classificadas como Tipo I podem ser chamadas de Excelentes, segundo Marques e Braga (1995), devido ao alto nível de liquidez praticado. Nesse caso, Araújo et al. (2013) destacam que tanto o NCG quanto o CDG da empresa podem ser entendidos como fontes de recursos, enquanto o SDT é visto como uma aplicação. Já as estruturas financeiras de empresas classificadas como Tipo II podem ser chamadas de Sólidas. De acordo com Machado et al. (2006, p. 143), nessa classificação, "os recursos de longo prazo investidos no [CDG] garantirão a continuidade de um SDT favorável, desde que o nível de atividade operacional seja mantido".

A estrutura financeira das empresas categorizada como Tipo III pode ser considerada Insatisfatória. Conforme Vieira (2008), essa classificação é a mais frequentemente encontrada nas empresas brasileiras. Nessa classificação, a demanda operacional é financiada por meio da 
composição de recursos de longo prazo (CDG) e de curto prazo (SDT). Por sua vez, estruturas financeiras de empresas classificadas como Tipo IV são categorizadas como Péssimas. Marques e Braga (1995) ressaltam que o CDG negativo sinaliza que fontes de curto prazo financiam investimentos de longo prazo. Ademais, como há uma NCG positiva, emprega-se passivo oneroso de curto prazo para cobrir tal necessidade (MARQUES; BRAGA, 1995).

Estruturas financeiras de empresas classificadas como Tipo V estão em uma situação Muito Ruim. Nesse caso, segundo Vieira (2008), o financiamento de investimentos de longo prazo será realizado por meio de recursos originários das atividades operacionais e de instituições financeiras, ficando sujeitas a flutuações da conjuntura econômica, do mercado financeiro e das políticas de crédito bancárias. Por fim, estruturas financeiras de empresas classificadas como Tipo VI são chamadas de Alto Risco. Nesse sentido, Machado et al. (2006, p. 143) afirmam que

\begin{abstract}
[CDG] e NCG permanecem negativos, porém o valor da NCG é inferior ao do [CDG]. Essa circunstância permite que o [SD]T seja positivo e pode sinalizar para o fato de a empresa não estar desempenhando suas operações de maneira adequada. Esse tipo de estrutura indica que a empresa estaria desviando sobras de recursos de curto prazo para ativos não circulantes e mantendo um saldo positivo de tesouraria.
\end{abstract}

Naturalmente, o modelo dinâmico pode ser empregado para se analisar a gestão de capital de giro de OPSs brasileiras, com enfoque na sua estrutura financeira. Todavia, é importante destacar que as operadoras brasileiras são muito heterogêneas e apresentam características bastante distintas de acordo com a sua modalidade (GUIMARÃES; NOSSA, 2010; SOUZA et al., 2016). O Quadro 2 apresenta um resumo das demais modalidades segundo de OPSs segundo Almeida e Sant'Anna (2010). Verifica-se que as operadoras das diferentes modalidades apresentam características bem distintas entre si, tais como a finalidade lucrativa das Seguradoras de Saúde ou das classificadas como Medicina de Grupo em contraposição à finalidade não lucrativa das OPSs Filantrópicas e o foco nos cooperados das cooperativas médicas e odontológicas.

\begin{tabular}{|c|c|}
\hline Modalidade & Descrição \\
\hline Medicina de grupo & $\begin{array}{l}\text { Operam com um tipo de contrato popularmente conhecido como convênio médico. Refere-se } \\
\text { à prestação de serviços médicos e hospitalares mediante a realização de contratos com os } \\
\text { clientes (beneficiários) dos quais são cobrados valores fixos submetidos a reajustes } \\
\text { periódicos. }\end{array}$ \\
\hline $\begin{array}{l}\text { Cooperativa } \\
\text { médica }\end{array}$ & $\begin{array}{l}\text { Constituem a modalidade em que os médicos são simultaneamente sócios e prestadores de } \\
\text { serviços e recebem pagamento de forma proporcional à produção de cada um (isto é, por tipo } \\
\text { e quantidade de atendimento). }\end{array}$ \\
\hline Autogestão & $\begin{array}{l}\text { É o modelo em que a própria empresa ou organização administra o programa de assistência à } \\
\text { saúde dos seus empregados e dependentes. Pode ser operado com recursos e serviços } \\
\text { credenciados (convênios) ou de livre escolha (reembolso). Adicionalmente, as autogestões só } \\
\text { podem operar planos coletivos que sejam restritos a um grupo exclusivo de beneficiários. }\end{array}$ \\
\hline Filantropias & São entidades sem fins lucrativos que operam planos privados de assistência à saúde, \\
\hline
\end{tabular}


AVELAR, E. A.; SOUZA, A. A. de. Gestão do capital de giro em operadoras de planos de saúde: uma análise sob a perspectiva do modelo dinâmico

\begin{tabular}{|c|l|}
\hline & $\begin{array}{l}\text { certificadas como entidade filantrópica junto ao Conselho Nacional de Assistência Social } \\
\text { (CNAS) e declaradas de utilidade pública junto ao Ministério da Justiça ou junto aos órgãos } \\
\text { dos governos estaduais e municipais. }\end{array}$ \\
\hline Administradoras & $\begin{array}{l}\text { Empresas que coordenam, controlam e administram planos de assistência à saúde } \\
\text { financiados por outra OPS. }\end{array}$ \\
\hline $\begin{array}{c}\text { Seguradora esp. em } \\
\text { saúde }\end{array}$ & $\begin{array}{l}\text { A maior parte dessas empresas é vinculada a empresas independentes nacionais e a bancos } \\
\text { nacionais. }\end{array}$ \\
\hline
\end{tabular}

Quadro 2 - Resumo das referidas modalidades

Fonte: Adaptado de Almeida e Sant'Anna (2010).

Diversos estudos nacionais têm empregado o modelo dinâmico em sua análise na última década, tais como Francisco et al. (2012), Camargos et al. (2014) e Lima et al. (2015). Porém, no caso de OPSs, destaca-se apenas o estudo de Guimarães e Nossa (2010). Esses autores analisaram a estrutura financeira de algumas modalidades de OPSs brasileiras, com foco na relação dessa estrutura com a lucratividade, a liquidez e a solvência dessas organizações. De acordo com Guimarães e Nossa (2010), nesse sentido, as melhores estruturas financeiras seriam as seguintes, nesta ordem: (1) excelente; (2) sólida; (3) insatisfatória; (4) alto risco; (5) muito ruim; (6) péssima. Todavia, o referido estudo não enfocou a evolução dessas estruturas financeiras e abordou apenas duas modalidades de OPSs, quais sejam: Odontologia de Grupo e Cooperativa Odontológicas. Para ampliar a discussão dessa área, este estudo considera um maior número de OPSs e modalidades em relação à Guimarães e Nossa (2010), e relaciona essa variável (modalidade) às diferentes estruturas financeiras previstas pelo modelo dinâmico.

\section{METODOLOGIA}

A pesquisa cujos resultados são apresentados neste trabalho pode ser classificada como descritiva e quantitativa. Conforme Sampieri et al. (2006), pesquisas com enfoque quantitativo são aquelas que empregam técnicas que visam "testar hipóteses com base na medição numérica e na análise estatística para estabelecer padrões de comportamento”. Já a pesquisa descritiva, segundo Malhotra e Birks (2007), é aquela cujo principal objetivo é a descrição de um dado fenômeno.

A população se refere a todas as OPSs brasileiras. A amostra empregada, porém, consistiu nas OPSs que apresentaram seus dados financeiros anuais ao longo do período de estudo (2012 a 2016) publicamente no sítio da ANS. Salienta-se que, no estudo, não foram consideradas as operadoras da modalidade Administradora - por não assumirem os riscos das operações como as demais OPSs -, nem as seguradoras especializadas em saúde - por se apresentarem em um número bastante reduzido no país, segundo dados da ANS (2017). Assim, foram consideradas as operadoras classificadas nas seguintes modalidades: autogestão (AG), cooperativa médica (CM), 
AVELAR, E. A.; SOUZA, A. A. de. Gestão do capital de giro em operadoras de planos de saúde: uma análise sob a perspectiva do modelo dinâmico

cooperativa odontológica (CO), filantropia (FI), medicina de grupo (MG) e odontologia de grupo (OG).

Para o desenvolvimento da pesquisa, foram empregados essencialmente dados secundários. Estes são dados coletados previamente para determinado fim, mas que são utilizados posteriormente para estudos com fins diversos, tais como as demonstrações financeiras (COOPER; SCHINDLER, 2003). Foram coletados especialmente dados financeiros provenientes das demonstrações financeiras publicadas pelas organizações que foram analisadas.

Esses dados foram baixados em diferentes formatos diretamente do sítio da ANS. Posteriormente, os mesmos foram organizados e tabulados no Microsoft ${ }^{\circledR}$ Excel (MS-Excel). Todos os dados das contas foram padronizados de forma em um plano de contas padrão que atendesse aos objetivos do pesquisador. Para padronizar esse plano de contas, foram estudadas as seguintes normas emitidas pela ANS: IN 46, RN 290 e RN 314 (normas que alteraram substancialmente os planos de contas das operadoras no período de análise). Posteriormente, os dados foram usados para se calcular as variáveis do modelo dinâmico citadas na Figura 1. Em seguida, com base nas variáveis previamente calculadas, as estruturas financeiras foram classificadas conforme o Quadro 1.

Para a análise dos dados, foram empregadas as seguintes técnicas: estatística descritiva e a estatística do Qui-quadrado. Conforme Mann (2006, p. 3), a estatística descritiva "consiste em métodos para se organizar, exibir e descrever dados utilizando tabelas, gráficos e medidas resumidas". Esta técnica foi aplicada na análise dos dados das OPS por modalidade, e considerando sua estrutura financeira, tanto anualmente (análise transversal), quanto ao longo do período (análise longitudinal). Para tanto, empregaram-se tabulações cruzadas, ou seja, duas variáveis foram analisadas simultaneamente. Já a estatística do Qui-Quadrado, de acordo com Malhotra e Birks (2007), é um teste estatístico empregado para se analisar a significância de uma associação observada em uma tabulação cruzada. Na pesquisa ora apresentada, essa técnica visou analisar se há uma distribuição homogênea das diferentes estruturas financeiras do modelo dinâmico em relação às distintas modalidades de OPSs.

\section{RESULTADOS}

Esta seção apresenta e analisa os resultados obtidos na pesquisa empírica. A Figura 2 apresenta o número de OPSs em cada uma das estruturas financeiras do Modelo Dinâmico ao longo do período em análise. Verifica-se, que, na média, $89,1 \%$ das OPSs se classificaram em uma das seguintes estruturas financeiras: excelente, sólida ou alto risco. Essa situação pode ser relacionada à regulação da ANS que exige uma melhor situação financeira dessas operadoras. 
AVELAR, E. A.; SOUZA, A. A. de. Gestão do capital de giro em operadoras de planos de saúde: uma análise sob a perspectiva do modelo dinâmico

Figura 2 - Número de OPSs em cada uma das estruturas financeiras do Modelo Dinâmico

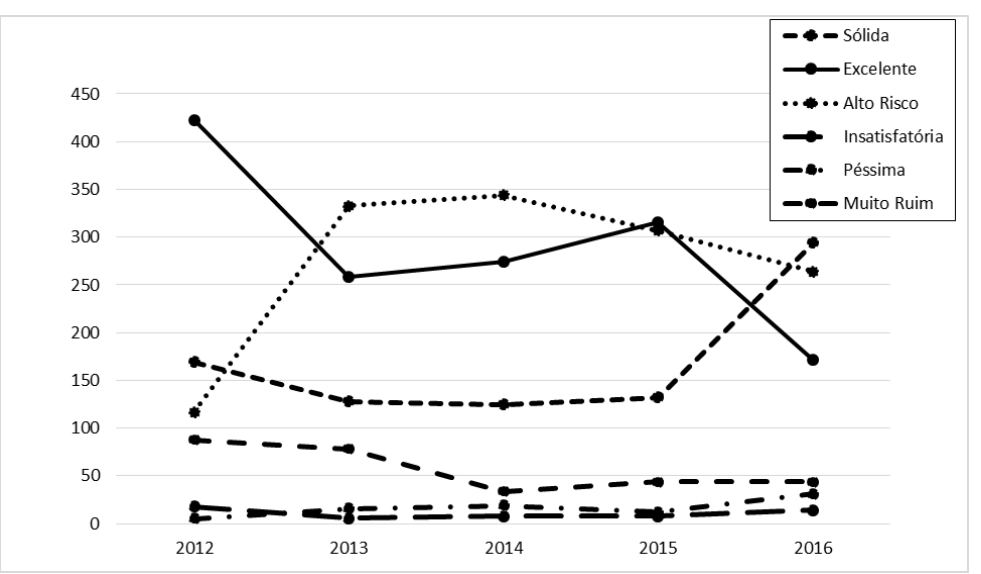

Fonte: Dados da pesquisa.

Ao se analisar a evolução das estruturas financeiras ao longo do tempo, verifica-se que houve uma queda significativa nas classificadas como excelentes. Em números absolutos, as OPSs que apresentaram essa estrutura financeira caíram de 422 de operadoras em 2012 para 172 em 2016. Já o número de OPSs classificadas como de alto risco aumentou de 117 em 2012 para 344 em 2014. A diferença entre as duas classificações supracitadas é a alteração no CDG: no caso das excelentes, esse valor é positivo; enquanto no caso das de alto risco, esse valor é negativo. Já no caso da estrutura financeira sólida, houve um aumento significativo nos últimos anos de estudo: em 2015, o número de OPSs era de 132; aumentando para 294 em 2016. Essa estrutura financeira é estreitamente relacionada às duas últimas: excelentes e alto risco. Uma redução do NCG levaria a OPS a se classificar como excelente. Já um aumento do NGC, aliado à uma redução do CDG, levaria a OPS a se classificar como alto risco.

Por sua vez, a Tabela 1 apresenta o número de OPSs segregado por sua modalidade e sua estrutura financeira para o ano de 2012. Neste ano, todas as modalidades de OPSs apresentaram uma maior frequência da estrutura financeira classificada como excelente: AG (45,0\%), CMs $(64,9 \%)$, COs (43,4\%), FI (35,3\%), MG (45,36\%) e OG (41,76\%). Apesar dessa situação, a estatística do Qui-quadrado demonstrou que há uma distribuição assimétrica entre as diferentes modalidades de OPSs e as diferentes estruturas financeiras. No caso das COs, 43,4\% das suas estruturas financeiras são classificadas como sólidas. No caso das OPSs filantrópicas, salienta-se que $23,5 \%$ das estruturas financeiras observadas são de alto risco. Já no caso das OPSs de OG, ressalta-se que $27,5 \%$ das estruturas financeiras são classificadas como muito ruim.

Tabela 1 - Número de OPSs segregado por sua modalidade e sua estrutura financeira para o ano de 2012

\begin{tabular}{|c|c|c|c|c|c|c|c|c|c|c|c|c|}
\hline \multirow{2}{*}{$\begin{array}{l}\text { Modalidade } \\
\text { Freq. }\end{array}$} & \multicolumn{2}{|c|}{$\mathbf{A G}$} & \multicolumn{2}{|c|}{$\mathbf{C M}$} & \multicolumn{2}{|c|}{ CO } & \multicolumn{2}{|c|}{ FI } & \multicolumn{2}{|c|}{ MG } & \multicolumn{2}{|c|}{ OG } \\
\hline & $\mathbf{n}$ & $\%$ & $\mathbf{n}$ & $\%$ & $\mathbf{n}$ & $\%$ & $\mathbf{n}$ & $\%$ & $\mathbf{n}$ & $\%$ & n & $\%$ \\
\hline Sólida & 29 & 30 & 40 & 14 & 43 & 43 & 8 & 16 & 34 & 19 & 15 & 16 \\
\hline Excelente & 48 & 49 & 192 & 65 & 43 & 43 & 18 & 35 & 83 & 45 & 38 & 42 \\
\hline Alto Risco & 12 & 12 & 44 & 15 & 3 & 3 & 12 & 24 & 34 & 19 & 12 & 13 \\
\hline Insatisfatória & 0 & - & 4 & 1 & 5 & 5 & 2 & 4 & 6 & 3 & 1 & 1 \\
\hline Péssima & 1 & 1 & 2 & 1 & 0 & - & 0 & - & 2 & 1 & 0 & - \\
\hline Muito Ruim & 8 & 8 & 14 & 5 & 5 & 5 & 11 & 22 & 24 & 13 & 25 & 27 \\
\hline Total & 98 & 100 & 296 & 100 & 99 & 100 & 51 & 100 & 183 & 100 & 91 & 100 \\
\hline
\end{tabular}

Fonte: Dados da pesquisa.

Notas: "n" equivale a frequência absoluta e "\%" a frequência relativa.

Estatística do Qui-quadrado: 125,2 (significativo a menos de 1,0\%) 
AVELAR, E. A.; SOUZA, A. A. de. Gestão do capital de giro em operadoras de planos de saúde: uma análise sob a perspectiva do modelo dinâmico

Já no caso da Tabela 2, é apresentado o número de OPSs segregado por sua modalidade e sua estrutura financeira para o ano de 2013. Nesse ano, verifica-se um grande aumento das OPSs cujas estruturas financeiras se classificam como de alto risco. Nesse caso, essa estrutura financeira se mostrou a com maior frequência nas seguintes modalidades: CMs $(52,7 \%)$, COs (24,2\%), FI (52,9\%) e MG (43,72\%). É interessante salientar o fato de o número de CMs classificadas como alto risco, cujo número de estruturas financeiras alterou em 700,0\% em relação ao ano anterior. Já as operadoras de AG e OG apresentaram estruturas financeiras excelentes em grande parte. Novamente, a estatística do Qui-quadrado apresentou uma distribuição assimétrica entre as distintas modalidades de OPSs e as diferentes estruturas financeiras.

Tabela 2 - Número de OPSs segregado por sua modalidade e sua estrutura financeira para o ano de 2013

\begin{tabular}{|c|c|c|c|c|c|c|c|c|c|c|c|c|}
\hline \multirow{2}{*}{$\begin{array}{l}\text { Modalidade } \\
\text { Freq. }\end{array}$} & \multicolumn{2}{|c|}{$\mathbf{A G}$} & \multicolumn{2}{|c|}{$\mathbf{C M}$} & \multicolumn{2}{|c|}{$\mathrm{CO}$} & \multicolumn{2}{|c|}{ FI } & \multicolumn{2}{|c|}{ MG } & \multicolumn{2}{|c|}{ OG } \\
\hline & $\mathbf{n}$ & $\%$ & $\mathbf{n}$ & $\%$ & $\mathbf{n}$ & $\%$ & $\mathbf{n}$ & $\%$ & n & $\%$ & $\mathbf{n}$ & $\%$ \\
\hline Sólida & 26 & 27 & 16 & 5 & 36 & 36 & 4 & 8 & 27 & 15 & 19 & 21 \\
\hline Excelente & 45 & 46 & 101 & 34 & 29 & 29 & 6 & 12 & 55 & 30 & 22 & 24 \\
\hline Alto Risco & 22 & 22 & 156 & 53 & 24 & 24 & 27 & 53 & 80 & 44 & 24 & 26 \\
\hline Insatisfatória & 0 & - & 1 & 0 & 0 & - & 0 & - & 2 & 1 & 3 & 3 \\
\hline Péssima & 1 & 1 & 4 & 1 & 3 & 3 & 3 & 6 & 3 & 2 & 2 & 2 \\
\hline Muito Ruim & 4 & 4 & 18 & 6 & 7 & 7 & 11 & 22 & 16 & 9 & 21 & 23 \\
\hline Total & 98 & 100 & 296 & 100 & 99 & 100 & 51 & 100 & 183 & 100 & 91 & 100 \\
\hline
\end{tabular}

Fonte: Dados da pesquisa.

Notas: "n" equivale a frequência absoluta e "\%" a frequência relativa.

Estatística do Qui-quadrado: 155,1 (significativo a menos de 1,0\%)

Por sua vez, a Tabela 3 apresenta o número de OPSs segregado por sua modalidade e sua estrutura financeira para o ano de 2014. Nesse caso, há novamente um predomínio das estruturas financeiras classificadas como Alto Risco nas diferentes modalidades: CMs (52,7\%), FI (51,0\%), MG (44,4\%) e OG (35,9\%). Já no caso de operadoras classificadas como AG e CO, a estrutura financeira mais frequente foi a excelente. Destaca-se que estatística do Qui-quadrado, também em 2014, apresentou uma distribuição assimétrica entre as diferentes modalidades de OPSs e as distintas estruturas financeiras.

Tabela 3 - Número de OPSs segregado por sua modalidade e sua estrutura financeira para o ano de 2014

\begin{tabular}{|c|c|c|c|c|c|c|c|c|c|c|c|c|}
\hline \multirow{2}{*}{$\begin{array}{l}\text { Modalidade } \\
\text { Freq. }\end{array}$} & \multicolumn{2}{|c|}{$\mathbf{A G}$} & \multicolumn{2}{|c|}{$\mathbf{C M}$} & \multicolumn{2}{|c|}{$\mathrm{CO}$} & \multicolumn{2}{|c|}{ FI } & \multicolumn{2}{|c|}{ MG } & \multicolumn{2}{|c|}{ OG } \\
\hline & $\mathbf{n}$ & $\%$ & $\mathbf{n}$ & $\%$ & $\mathbf{n}$ & $\%$ & $\mathbf{n}$ & $\%$ & $\mathbf{n}$ & $\%$ & $\mathbf{n}$ & $\%$ \\
\hline Sólida & 31 & 33 & 18 & 6 & 31 & 32 & 3 & 6 & 24 & 13 & 18 & 21 \\
\hline Excelente & 41 & 44 & 106 & 36 & 34 & 35 & 6 & 12 & 59 & 33 & 28 & 33 \\
\hline Alto Risco & 21 & 22 & 161 & 55 & 26 & 27 & 26 & 51 & 80 & 44 & 30 & 35 \\
\hline Insatisfatória & 0 & - & 0 & - & 2 & 2 & 0 & - & 1 & 1 & 5 & 6 \\
\hline Péssima & 1 & 1 & 4 & 1 & 4 & 4 & 4 & 8 & 5 & 3 & 1 & 1 \\
\hline Muito Ruim & 0 & - & 5 & 2 & 1 & 1 & 12 & 24 & 11 & 6 & 4 & 5 \\
\hline Total & 94 & 100 & 294 & 100 & 98 & 100 & 51 & 100 & 180 & 100 & 86 & 100 \\
\hline
\end{tabular}

O número de OPSs segregado por sua modalidade e sua estrutura financeira para o ano de 2015 é apresentado na Tabela 4. Nesse caso, observa-se que a estrutura financeira excelente foi a ForSci.: r. cient. IFMG campus Formiga, Formiga, v. 6 , n. 1, e00300, 2018. 
mais frequente nas seguintes modalidades de operadoras: AG (42,9\%), CO (42,4\%) e MG $(38,3 \%)$. Já a estrutura financeira alto risco foi a mais observada nas seguintes modalidades de OPS: CMs (46,3\%), FI (64,7\%), e OG (35,1\%). Salienta-se que, apesar da estrutura mais frequente ser a de alto risco no caso das CMs, houve um aumento significativo no caso de estruturas financeiras excelentes. Já no caso das operadoras filantrópicas, em 2015, houve o maior número de estruturas financeiras de alto risco. A estatística do Qui-quadrado também apresentou uma distribuição assimétrica entre as distintas modalidades de OPSs e as diferentes estruturas financeiras.

Tabela 4 - Número de OPSs segregado por sua modalidade e sua estrutura financeira para o ano de 2015

\begin{tabular}{|c|c|c|c|c|c|c|c|c|c|c|c|c|}
\hline \multirow{2}{*}{$\begin{array}{l}\text { Modalidade } \\
\text { Freq. }\end{array}$} & \multicolumn{2}{|c|}{$\mathbf{A G}$} & \multicolumn{2}{|c|}{ CM } & \multicolumn{2}{|c|}{$\mathrm{CO}$} & \multicolumn{2}{|c|}{ FI } & \multicolumn{2}{|c|}{ MG } & \multicolumn{2}{|c|}{$\mathbf{O G}$} \\
\hline & $\mathbf{n}$ & $\%$ & n & $\%$ & $\mathbf{N}$ & $\%$ & $\mathbf{n}$ & $\%$ & n & $\%$ & $\mathbf{n}$ & $\%$ \\
\hline Sólida & 31 & 32 & 20 & 7 & 27 & 27 & 3 & 6 & 33 & 18 & 18 & 20 \\
\hline Excelente & 42 & 43 & 127 & 43 & 42 & 42 & 6 & 12 & 70 & 38 & 29 & 32 \\
\hline Alto Risco & 19 & 19 & 137 & 46 & 20 & 20 & 33 & 65 & 65 & 36 & 32 & 35 \\
\hline Insatisfatória & 1 & 1 & 1 & 0 & 3 & 3 & 0 & - & 0 & - & 3 & 3 \\
\hline Péssima & 1 & 1 & 5 & 2 & 2 & 2 & 0 & - & 3 & 2 & 1 & 1 \\
\hline Muito Ruim & 4 & 4 & 6 & 2 & 5 & 5 & 9 & 18 & 12 & 7 & 8 & 9 \\
\hline Total & 98 & 100 & 296 & 100 & 99 & 100 & 51 & 100 & 183 & 100 & 91 & 100 \\
\hline
\end{tabular}

Por fim, na Tabela 5, apresenta o número de OPSs segregado por modalidade e sua estrutura financeira para o ano de 2016. Nesse ano, especificamente, são apresentadas as maiores frequências de estruturas financeiras sólidas para as seguintes modalidades de operadoras: AG $(58,1 \%)$, CO $(55,6 \%)$ e MG $(32,2 \%)$. Verifica-se uma redução da qualidade da estrutura financeira dessas OPSs, segundo Guimarães e Nossa (2010). Houve uma maior frequência da estrutura financeira de Alto Risco no caso das seguintes modalidades de operadoras: CMs $(41,2 \%)$, FI $(49,0 \%)$, e OG $(38,5 \%)$. Salienta-se o maior número de estruturas financeiras consideradas péssimas, em especial, nas modalidades CMs e MG. A estatística do Qui-quadrado novamente apresentou uma distribuição assimétrica entre as modalidades de OPSs e as diferentes estruturas financeiras.

Tabela 5 - Número de OPSs segregado por sua modalidade e sua estrutura financeira para o ano de 2016

\begin{tabular}{|c|c|c|c|c|c|c|c|c|c|c|c|c|}
\hline \multirow{2}{*}{$\begin{array}{l}\text { Modalidade } \\
\text { Freq. }\end{array}$} & \multicolumn{2}{|c|}{$\mathbf{A G}$} & \multicolumn{2}{|c|}{$\mathbf{C M}$} & \multicolumn{2}{|c|}{ CO } & \multicolumn{2}{|c|}{ FI } & \multicolumn{2}{|c|}{ MG } & \multicolumn{2}{|c|}{ OG } \\
\hline & n & $\%$ & $\mathbf{n}$ & $\%$ & $\mathbf{n}$ & $\%$ & $\mathbf{n}$ & $\%$ & n & $\%$ & $\mathbf{n}$ & $\%$ \\
\hline Sólida & 57 & 58 & 97 & 33 & 55 & 56 & 6 & 12 & 59 & 32 & 20 & 22 \\
\hline Excelente & 26 & 27 & 53 & 18 & 19 & 19 & 4 & 8 & 48 & 26 & 22 & 24 \\
\hline Alto Risco & 14 & 14 & 122 & 41 & 15 & 15 & 25 & 49 & 53 & 29 & 35 & 38 \\
\hline Insatisfatória & 0 & - & 5 & 2 & 1 & 1 & 0 & - & 3 & 2 & 5 & 5 \\
\hline Péssima & 1 & 1 & 12 & 4 & 6 & 6 & 1 & 2 & 9 & 5 & 2 & 2 \\
\hline Muito Ruim & 0 & - & 7 & 2 & 3 & 3 & 15 & 29 & 11 & 6 & 7 & 8 \\
\hline Total & 98 & 100 & 296 & 100 & 99 & 100 & 51 & 100 & 183 & 100 & 91 & 100 \\
\hline
\end{tabular}

Fonte: Dados da pesquisa.

Notas: " $n$ " equivale a frequência absoluta e "\%" a frequência relativa.

Estatística do Qui-quadrado: 165,2 (significativo a menos de 1,0\%) 
Os resultados obtidos foram resumidos no Quadro 3. Dentre as modalidades analisadas, as OPSs classificadas como AG apresentaram as melhores estruturas financeiras ao longo do período estudado. As COs também apresentaram, em geral, estruturas financeiras mais favoráveis. Já no caso das CMs e MGs, as operadoras apresentaram uma deterioração das estruturas financeiras em geral. As operadoras filantrópicas foram as que apresentaram as piores estruturas financeiras de uma forma geral (alto risco) ao longo do período de análise.

\begin{tabular}{|c|c|c|c|c|c|}
\hline \multirow{2}{*}{ Modalidade } & \multicolumn{5}{|c|}{ Estrutura financeira mais frequente } \\
\cline { 2 - 6 } & $\mathbf{2 0 1 2}$ & $\mathbf{2 0 1 3}$ & $\mathbf{2 0 1 4}$ & $\mathbf{2 0 1 5}$ & $\mathbf{2 0 1 6}$ \\
\hline AG & Excelente & Excelente & Excelente & Excelente & Sólida \\
\hline CM & Excelente & Alto Risco & Alto Risco & Alto Risco & Alto Risco \\
\hline CO & Excelente & Alto Risco & Excelente & Excelente & Sólida \\
\hline FI & Excelente & Alto Risco & Alto Risco & Alto Risco & Alto Risco \\
\hline MG & Excelente & Alto Risco & Alto Risco & Excelente & Sólida \\
\hline OG & Excelente & Excelente & Alto Risco & Alto Risco & Alto Risco \\
\hline
\end{tabular}

Quadro 3 - Estruturas financeiras mais frequentes por modalidade de OPS entre 2012 e 2016 Fonte: Dados da pesquisa.

Uma análise mais detalhada do Quadro 3 indica que as operadoras da modalidade AG apresentaram uma redução do NCG ao longo do tempo, deixando de ser uma fonte de recursos para se tornar uma aplicação de recursos (nesse caso, oriundos do CDG). Já no caso das operadoras classificadas como CM e FI, a partir de 2013, houve uma queda em seus valores de CDG, sendo que o financiamento deste e do SDT passou a ser realizado por meio do NCG (indicando o alto risco desta estrutura financeira). Para as COs, apesar de uma queda na qualidade de sua estrutura financeira em 2013 (semelhante à ocorrida com as CMs), houve uma melhora em seu CDG em 2014, que passou a financiar novamente tanto o SDT quanto a NCG. Nas MGs, ocorreu um maior número de variações ao longo do tempo devido às variações do CDG e do NCG, todavia, o aumento daquela variável no final do período conseguiu garantir uma estrutura financeira sólida a essas organizações. Por fim, a queda do CDG das OGs tornou essas organizações dependentes do seu NCG de tal forma que aumentou significativamente o risco de sua estrutura financeira.

\section{CONCLUSÕES}

Os resultados obtidos na pesquisa empírica demonstram que as estruturas financeiras das OPSs, que evidenciam aspectos da gestão de seu capital de giro segundo o modelo dinâmico, são discrepantes entre as diferentes modalidades de operadoras. Tal observação realça a heterogeneidade do desempenho dessas organizações, como destacado por Souza et al. (2016). Tal constatação foi estatisticamente significativa em todos os anos analisados, conforme a estatística do Qui-quadrado. No caso das operadoras classificadas como AG, observaram-se estruturas financeiras predominantemente excelentes ao longo do período de análise. Nesse caso, segundo Araújo et al. (2013), tanto a NCG quanto o CDG são fontes de recursos para a empresa, 
que pode aplicar os recursos excedentes no mercado financeiro. Um resultado próximo foi observado no caso das COs, todavia, verificou-se um comportamento mais errático de suas estruturas financeiras ao longo do tempo.

Já no caso das operadoras das modalidades de CMs, das FIs e das OGs, constatou-se que, em geral, as mesmas apresentaram estruturas financeiras de alto risco ao longo do período de análise. Nesse caso, há uma dependência do NCG para financiar tanto o CDG quanto o SDT das operadoras. Essa situação, de acordo com Machado et al. (2006), pode indicar o desempenho inadequado das atividades das mesmas. Por sua vez, aquelas classificadas como MG apresentaram uma deterioração geral de suas estruturas financeiras ao longo do período, mas melhoraram ao final da análise.

Considerando o exposto por Guimarães e Nossa (2010), sob a perspectiva da lucratividade, liquidez e solvência, pode-se classificar, de maneira geral, que a qualidade da gestão do capital de giro das operadoras, por modalidade, na seguinte ordem: (1) AG; (2) CO; (3) MG; (4) OGs; (5) CM; e (6) FI. É importante ressaltar que a estrutura financeira insatisfatória, considerada por Vieira (2008) a estrutura mais comum nas empresas brasileiras, não foi frequente entre as OPSs.

Apesar dessas constatações por modalidade, é importante ressaltar que, no geral, as operadoras de todas as modalidades apresentaram uma deterioração de suas estruturas financeiras entre os anos de 2015 e 2016. A alteração geral da estrutura excelente para sólida indica um aumento da demanda por capital de giro nessas organizações. Estudos futuros poderiam analisar os possíveis efeitos da regulação sobre os resultados ora observados. Além disso, variáveis endógenas como porte e nível de especialização das operadoras também poderiam ser discutidos futuramente.

Salientam-se algumas limitações do estudo apresentado neste artigo, tais como a desconsideração de outras variáveis para se mensurar a gestão do capital de giro (tais como indicadores de liquidez tradicionais) e o baixo número de observações em algumas estruturas financeiras de algumas modalidades (o que prejudica a qualidade da estatística do qui-quadrado). Apesar das limitações deste estudo, pode-se dizer que a pesquisa apresentada contribui de forma significativa para a discussão sobre a gestão do capital de giro das OPSs, que são organizações essenciais para o sistema de saúde nacional e extremamente reguladas pela ANS, com foco em seu aspecto econômico-financeiro.

\title{
WORKING CAPITAL MANAGEMENT IN HEALTHCARE INSURANCE COMPANIES: AN ANAL YSIS FROM THE PERSPECTIVE OF DYNAMIC MODEL
}

\begin{abstract}
This paper presents the results of a research that aimed to analyze the working capital management of Brazilian healthcare insurance companies (HICs) from the perspective of dynamic model. This study can be classified as a quantitative and descriptive one. We used data from 818 HICs from 2012 to 2016. These HICs are classified in different types according to the ForSci.: r. cient. IFMG campus Formiga, Formiga, v. 6, n. 1 , e00300, 2018.
\end{abstract}


national Agency of Supplementary Health (ANS): self-management, medical cooperative, dental cooperative, philanthropy, medicine of group and dentistry of group. The data were analyzed by the following techniques: descriptive statistics and chi-squared statistic. The empirical research shows that financing structures of HICs are different in according to their types (classification of ANS). This difference is considered significant by chi-squared statistic along all the analyzed period. Although these differences exist, it is important to note that the HICs of all types usually presented a deterioration of their financing structures between 2015 and 2016. In this case, it was verified an increase on working capital necessity.

Keywords: Working capital management. Dynamic model. Healthcare insurance. Financing structure.

\section{REFERÊNCIAS}

\section{AGÊNCIA NACIONAL DE SAÚDE SUPLEMENTAR - ANS. Resolução de Diretoria} Colegiada - RDC no 39, de 27 de outubro de 2000. 2000. Disponível em: <http://www. ans.gov.br/component/legislacao/?view=legislacao\&task=TextoLei \&format=raw\&id=Mzgw $>$. Acesso em: 06 dez. 2015.

Sala de Situação. 2017. Disponível em: <http://www.ans.gov.br/perfil-do-setor/dadose-indicadores-do-setor/sala-de-situacao>. Acesso em: 10 nov. 2017.

ALMEIDA, R. G.; SANT'ANNA, A. P. Composição Probabilística na Avaliação do Risco de Operadoras de Planos de Assistência à Saúde. Revista Brasileira de Risco e Seguro, v. 6, n. 11, p. 1-34, 2010.

AMBROZINI, M. A.; MATIAS, A. B.; PIMENTA JUNIOR, T. Análise dinâmica de capital de giro segundo o Modelo Fleuriet: uma classificação das empresas brasileiras de capital aberto no período de 1996 a 2013. Revista Contabilidade Vista \& Revista, v. 25, n. 2, p. 15-37, maio/ago, 2014.

ARAÚJO, E. A. T.; COSTA, M. L. O.; CAMARGOS, M. A. Mapeamento da produção científica sobre o Modelo Fleuriet no Brasil. Gestão Contemporânea, Porto Alegre, v. 10, n. 14, p. 311$347,2013$.

BRAGA, R. Análise avançada do capital de giro. Caderno de Estudos, n. 3, São Paulo, p. 1-20, 1991.

CAMARGOS, M. A.; CAMARGOS, M. C. S.; LEÃO, L. C. G. Empirically testing the "Fleuriet's model": Evidences of Brazilian Market. Business and Management Review, v. 4, n. 3, p. 165-177, 2014.

CARDOSO, R. L. Regulação econômica e escolhas de práticas contábeis: evidências no mercado de saúde suplementar brasileiro. 2005. 154 f. Tese (Doutorado em Ciências Contábeis) Universidade de São Paulo, São Paulo, 2005. Disponível em: <http://www.teses.usp.br/teses/ disponiveis/12/12136/tde-18122008-121952/pt-br.php>. Acesso em: 13 jan. 2017.

COOPER, D. R.; SCHINDLER, P. S. Métodos de pesquisa em administração. 7.ed. Porto Alegre: Bookman, 2003. 
COSTA, N. R. O regime regulatório e o mercado de planos de saúde no Brasil. Ciência \& Saúde Coletiva, v. 13, n. 5, p. 1453-1462, 2008.

ENGBERG, J. et al. The effect of mergers on firms' costs: evidence from the HMO industry. The Quarterly Review of Economics and Finance, v. 44, p. 574-600, 2004. Disponível em: <https://www.sciencedirect.com/science/article/abs/pii/S1062976903000358>. Acesso em: 10 jan. 2017.

FLEURIET, M. Fleuriet's Rebuttal to “Questioning Fleuriet's Model of Working Capital Management on Empirical Grounds. Social Science Research Network. 2005. Disponível em: <http://papers.ssrn.com/sol3/papers.cfm?abstract_id=741624>. Acesso em: 15 jul. 2016.

FLEURIET, M.; KEHDY, R.; BLANC, G. O modelo Fleuriet, a dinâmica financeira das empresas brasileiras: um modelo de análise, orçamento e planejamento financeiro. 5. ed. Rio de Janeiro: Elsevier, 2003

FRANCISCO, J. R. et al. Gestão Financeira do Segmento Bancos como Processo de Tomada de Decisão: Aplicação do Modelo Dinâmico. Pensar Contábil, v. 14, n. 55, p. 41-51, 2012.

GITMAN, L. J. Princípios de administração financeira. 12. ed. São Paulo: Pearson, 2010.

GUIMARÃES, A. L. S.; NOSSA, V. Working capital, profitability, liquidity and solvency of healthcare insurance companies. Brazilian Business Review, v. 7, n. 2, p. 37-59, 2010. Disponível em: <http://bbronline.com.br/public/edicoes/7_2/artigos/py8dy9ojrr2682016 154951.pdf >. Acesso em: 30 jun. 2017.

LIMA, C. A. et al. Análise financeira sob a luz do modelo dinâmico: um estudo no setor de telefonia fixa brasileira listado na Bovespa no período de 2008 a 2013. Revista Eletrônica de Administração, v. 14, n. 2, 2015. Disponível em: <http://periodicos.unifacef.com.br/index. php/rea/article/view/1010>. Acesso em: 10 jul. 2017.

MACHADO, M. A. V.; MACHADO, M. R.; CALLADO, A. L. C. Análise dinâmica e o financiamento das necessidades de capital de giro das pequenas e médias empresas localizadas na cidade de João Pessoa, PB: um estudo exploratório. BASE - Revista de Administração e Contabilidade da Unisinos, v. 3, n. 2, p. 139-149, 2006.

MAGALHÃES, R. S. et al. A utilidade da informação contábil no processo de físcalização e controle das operadoras de plano de saúde pela Agência Nacional de Saúde Suplementar (ANS). Revista Ambiente Contábil, v. 7, n. 2, p. 53-75, 2015.

MALHOTRA, N. K.; BIRKS, D. F. Marketing Research. 3 ed. Harlow: Prentice Hall, 2007.

MANN, Prem S. Introdução à estatística. 5 ed. Rio de Janeiro: LTC, 2006.

MARQUES, J. A. V. C.; BRAGA, R. Análise dinâmica do capital de giro: o modelo dinâmico. Revista de Administração de Empresas, v. 35, n. 3, p. 49-63, 1995.

PADOVEZE, C. L.; BENEDICTO, G. C. Análise das demonstrações financeiras. 3. ed. São Paulo: Cengace Learning, 2014. 
SAMPIERI, R. H.; COLLADO, C. H.; LUCIO, P. B. Metodologia de pesquisa. 3. ed. São Paulo: MacGraw-Hill, 2006.

SANCOVSCHI, M.; MACEDO, M. A. S.; SILVA, J. A. Análise das Intervenções da Agência Nacional de Saúde Suplementar (ANS) Através dos Índices de Desempenho da Saúde Suplementar (IDSS). Contabilidade, Gestão e Governança, Brasília, v. 17, n. 2, p. 118-136, 2014.

SOUZA, A. A. et al. Analysis of operating performance of Brazilian health plan. Systems \& Management, v. 11, n. 2, p. 175-182, 2016. Disponível em: <http://www.revistasg.uff.br/ index.php/sg/article/viewFile/818/478>. Acesso em: 30 jun. 2017.

THEISS JÚNIOR, F. C.; WILHELM, P. P. H. Análise do capital de giro: modelo dinâmico versus modelo tradicional. Revista de Gestão de Negócios, v. 5, n. 3, p. 1-11, 2000.

UKAEGBU, B. The significance of working capital management in determining firm profitability: Evidence from developing economies in Africa. Research in International Business and Finance, v. 31, p. 1-16, 2014. Disponível em: <https://www.sciencedirect. com/science/article/pii/S0275531913000779>. Acesso em: 01 jul. 2017.

VARELLA, D.; CESCHIN, M. A saúde dos planos de saúde: os desafios da assistência privada no Brasil. São Paulo: Paralela, 2014.

VIEIRA, M. V. Administração estratégica do capital de giro. 2. ed. São Paulo: Atlas, 2008.

\section{DADOS DOS AUTORES}

\section{Ewerton Alex Avelar}

Lattes: http://lattes.cnpq.br/6758338709153591

E-mail: ewertonalexavelar@gmail.com

Mestre em Administração pela Universidade Federal de Lavras (UFLA). É doutorando em Administração pela UFMG e atualmente é servidor da mesma instituição.

\section{Antônio Artur de Souza}

Lattes: http://lattes.cnpq.br/0597505816212353

E-mail: antonioarturdesouza@gmail.com

Ph.D. em Management Science pela Universidade de Lancaster (Reino Unido). Mestre em Engenharia de Produção pela Universidade Federal de Santa Catarina e atualmente é servidor da UFMG. 